\title{
Whole-genome comparative analysis at the lineage/sublineage level discloses relationships between Mycobacterium tuberculosis genotype and clinical phenotype
}

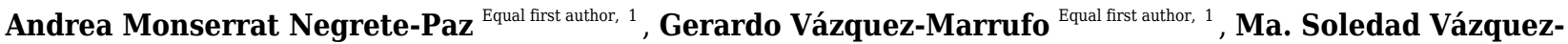 \\ Garcidueñas ${ }^{\text {Corresp. } 2}$ \\ ${ }^{1}$ Centro Multidisciplinario de Estudios en Biotecnología, Facultad de Medicina Veterinaria y Zootecnia, Universidad Michoacana de San Nicolás de Hidalgo, \\ Tarímbaro, Michoacán, Mexico \\ 2 División de Estudios de Posgrado, Facultad de Ciencias Médicas y Biológicas "Dr. Ignacio Chávez", Universidad Michoacana de San Nicolás de Hidalgo, \\ Morelia, Michoacán, México \\ Corresponding Author: Ma. Soledad Vázquez-Garcidueñas \\ Email address: soledad.vazquez@umich.mx
}

Background. Human tuberculosis (TB) caused by members of the Mycobacterium tuberculosis complex (MTBC) is the main cause of death among infectious diseases worldwide. Pulmonary TB (PTB) is the most common clinical phenotype of the disease, but some patients develop an extrapulmonary (EPTB) phenotype in which any organ or tissue can be affected. MTBC species include nine phylogenetic lineages, with some appearing globally and others being geographically restricted. EPTB can or not have pulmonary involvement, challenging its diagnosis when lungs are not implicated, thus causing an inadequate treatment. Finding evidence of a specific $M$. tuberculosis genetic background associated with EPTB is epidemiologically relevant due to the virulent and multidrugresistant strains isolated from such cases. Until now, the studies conducted to establish associations between $M$. tuberculosis lineages and PTB/EPTB phenotypes have shown inconsistent results, which are attributed to the strain predominance from specific $M$. tuberculosis lineages/sublineages in the samples analyzed and the use of low-resolution phylogenetic tools that have impaired sublineage discrimination abilities. The present work elucidates the relationships between the MTBC strain lineages/sublineages and the clinical phenotypes of the disease as well as the antibiotic resistance of the strains. Methods. To avoid biases, we retrieved the raw genomic reads (RGRs) of all $(n=245)$ the M. tuberculosis strains worldwide causing EPTB available in databases and an equally representative sample of the RGRs $(n=245)$ of PTB strains. A multiple alignment was constructed, and a robust maximum likelihood phylogeny based on single-nucleotide polymorphisms was generated, allowing effective strain lineage/sublineage assignment. Results. A significant Odds Ratio (OR range: 1.8-8.1) association was found between EPTB and the 1.1.1, 1.2.1, 4.1.2.1 and ancestral Beijing sublineages. Additionally, a significant association between 
PTB with 4.3.1, 4.3.3, and 4.5 and Asian African 2 and Europe/Russia B0/W148 modern Beijing sublineages was found. We also observed a significant association of Lineage 3 strains with multidrug resistance (OR 3.8; $95 \% \mathrm{Cl} 1.1-13.6)$, as well as between modern Beijing sublineages and antibiotic resistance (OR 4.3; 3.8-8.6). In this work, it was found that intralineage diversity can drive differences in the immune response that triggers the PTB/EPTB phenotype. 


\section{Whole-genome comparative analysis at the}

2 lineage/sublineage level discloses relationships

3 between Mycobacterium tuberculosis genotype and

4 clinical phenotype

Andrea Monserrat Negrete-Paz ${ }^{1}$, Gerardo Vázquez-Marrufo ${ }^{1}$, Ma. Soledad Vázquez-

7 Garcidueñas $^{2 *}$

8

${ }^{1}$ Centro Multidisciplinario de Estudios en Biotecnología, Facultad de Medicina Veterinaria y Zootecnia, Universidad Michoacana de San Nicolás de Hidalgo, Tarímbaro, Michoacán, Mexico

${ }^{2}$ División de Estudios de Posgrado, Facultad de Ciencias Médicas y Biológicas "Dr. Ignacio Chávez", Universidad Michoacana de San Nicolás de Hidalgo, Morelia, Michoacán, Mexico

*Corresponding Author:

Ma. Soledad Vázquez-Garcidueñas ${ }^{2}$

Ave. Rafael Carrillo esq. Dr. Salvador González Herrejón S/N, Col. Cuauhtémoc

C.P. 58020, Morelia, Michoacán, Mexico

E-mail address: soledad.vazquez@umich.mx

19

20

21

22

23

24

25

26

27

\section{Abstract}

Background. Human tuberculosis (TB) caused by members of the Mycobacterium tuberculosis complex (MTBC) is the main cause of death among infectious diseases worldwide. Pulmonary TB (PTB) is the most common clinical phenotype of the disease, but some patients develop an extrapulmonary (EPTB) phenotype in which any organ or tissue can be affected. MTBC species include nine phylogenetic lineages, with some appearing globally and others being geographically restricted. EPTB can or not have pulmonary involvement, challenging its diagnosis when lungs are not implicated, thus causing an inadequate treatment. Finding evidence of a specific M. tuberculosis genetic background associated with EPTB is epidemiologically relevant due to the virulent and multidrug-resistant strains isolated from such cases. Until now, the studies conducted to establish associations between M. tuberculosis lineages and PTB/EPTB phenotypes have shown inconsistent results, which are attributed to the strain predominance from specific M. tuberculosis lineages/sublineages in the samples analyzed and the use of lowresolution phylogenetic tools that have impaired sublineage discrimination abilities. The present work elucidates the relationships between the MTBC strain lineages/sublineages and the clinical phenotypes of the disease as well as the antibiotic resistance of the strains.

Methods. To avoid biases, we retrieved the raw genomic reads (RGRs) of all ( $\mathrm{n}=245)$ the $M$. tuberculosis strains worldwide causing EPTB available in databases and an equally 
representative sample of the RGRs ( $\mathrm{n}=245)$ of PTB strains. A multiple alignment was constructed, and a robust maximum likelihood phylogeny based on single-nucleotide polymorphisms was generated, allowing effective strain lineage/sublineage assignment.

Results. A significant Odds Ratio (OR range: 1.8-8.1) association was found between EPTB and the 1.1.1, 1.2.1, 4.1.2.1 and ancestral Beijing sublineages. Additionally, a significant association between PTB with 4.3.1, 4.3.3, and 4.5 and Asian African 2 and Europe/Russia B0/W148 modern Beijing sublineages was found. We also observed a significant association of Lineage 3 strains with multidrug resistance (OR 3.8; 95\% CI 1.1-13.6), as well as between modern Beijing sublineages and antibiotic resistance (OR 4.3; 3.8-8.6). In this work, it was found that intralineage diversity can drive differences in the immune response that triggers the PTB/EPTB phenotype.

\section{Introduction}

Tuberculosis (TB) represents the main cause of death among infectious diseases worldwide, with its drug-resistant manifestations constituting a major global health concern (Floyd et al., 2018). Pulmonary TB (PTB) is the most common clinical disease phenotype, but some patients develop an extrapulmonary TB (EPTB) phenotype in which practically any organ or tissue can be affected, including the aggressive manifestations of lymph node and central nervous system TB (Golden \& Vikram, 2005). EPTB represents approximately $15 \%$ of reported TB cases globally, whereas as many as $40 \%$ of TB cases in several high-income countries are EPTB (WHO, 2020). Human TB is caused by members of the Mycobacterium tuberculosis complex (MTBC), which have $>99 \%$ nucleotide sequence identity at the genomic level (Gagneux, 2018). The humanadapted species of the MTBC are M. tuberculosis sensu stricto and Mycobacterium africanum, which are divided into nine phylogenetic lineages: L1, or Indo-Oceanic; L2, or East Asian; L3, or East African-Indian; L4, or Euro-American; L5, or M. africanum West-African 1; L6, or M. africanum West-African 2; L7, or Ethiopia (Firdessa et al., 2013; Coscolla \& Gagneux, 2014); L8, or M. tuberculosis from the African Great Lakes (Ngabonziza et al., 2020); and the recently described M. africanum L9 (Coscolla et al., 2021). Global phylogeographic reconstruction of $M$. tuberculosis suggests that each lineage has become specifically adapted to defined human populations (Gagneux, 2018), with some occurring globally and others showing strong geographical restriction. L4, L2, and L3 are the most commonly found lineages; nevertheless, L4 is the most widespread lineage worldwide. L3 is mostly found in the Middle East, India, and East Africa, while L2 is found predominantly in East Asia (McHenry et al., 2020). This geographical restriction has been found even at the sublineage level, as in sublineage 4.6/Uganda, found only in Uganda and neighboring countries. This sublineage has been shown to possess highly conserved $\mathrm{T}$ cell epitopes and a restricted geographic distribution, suggesting a possible adaptation to a specific human population (Stucki et al., 2016). On the other hand, the high incidence of infections associated with nongeographically restricted strains might imply that these strains are more effective in causing the disease (Malik \& Godfrey-Fausset, 2005). 
79

80

81

82

83

84

85

86

87

88

89

90

91

92

93

94

95

96

97

98

99

100

101

102

103

104

105

106

107

108

109

110

111

112

113

114

115

116

117

118

Several studies have been conducted to identify possible relationships between $M$. tuberculosis phylogenetic lineages and the PTB or EPTB phenotype of the disease (Feng et al., 2008; Click et al., 2012), but the results show a lack of consistency. Different factors contribute to explaining the observed discrepancies among the conducted studies in an attempt to establish genotype-phenotype relationships. In the first instance, there were differences in the sample size (Coscolla \& Gagneux, 2014), as well as in the nonhomogeneous distribution of lineages, among the set (Kato-Maeda \& Nahid, 2012) of analyzed strains. Moreover, biased associations might arise due to a lack of data or failure to control for possible confounders associated with known risk factors for EPTB, such as human immunodeficiency virus (HIV) infection comorbidity in patients from whom strains were isolated (Coscolla \& Gagneux, 2014). Furthermore, studies use different operational definitions for EPTB (Kato-Maeda \& Nahid, 2012), and some lack appropriate tools to index genomic diversity and classify strains into lineages in some studies (Coscolla \& Gagneux, 2010). Whole-genome comparative analysis has allowed the categorization of M. tuberculosis lineages into sublineages using single-nucleotide polymorphism (SNP) analysis (Coll et al., 2014; Stucki et al., 2016). This subtle level of strain differentiation suggests that some sublineages might drive the observed associations of an $M$. tuberculosis genotype with a specific disease phenotype, such as EPTB (Feng et al., 2008), but an analysis to provide evidence in support of this hypothesis has not been conducted. Interestingly, the frequency of the sublineages assigned to isolated strains from the East Asian (L2) lineage differs among populations settled in different geographical areas, which might also explain why some studies associate L2 lineage strains with EPTB (Feng et al., 2008), whereas others associate it with PTB (Dale et al., 2005), and still others do not find any association at all (Svensson et al., 2011). Thus, intralineage diversity requires a detailed exploration to clarify disease phenotype variation and its relationship with MTBC genotypes (Kato-Maeda \& Nahid, 2012). Additionally, genomic evidence has revealed a close relationship among specific $M$. tuberculosis lineages and sublineages with drug resistance (Wang et al., 2015), a relevant public health phenotype that might hinder successful TB treatment. Horizontal transfer of drug resistance genes has not been reported for $M$. tuberculosis, but resistance mostly arises from chromosomal mutations under the selective pressure of antibiotic use (Nguyen, 2016). Thus, the characterization of mutations associated with resistance phenotypes in strains with different genotypes can help to reveal lineage-/sublineage-specific microevolutionary processes of epidemiological relevance. Drug-resistance-associated mutations have been hypothesized to modify strain fitness and the ability of a strain to cross the blood-brain barrier, causing EPTB, specifically tuberculous meningitis (Faksri et al., 2018).

Unclarified relationships of lineages/sublineages with M. tuberculosis PTB/EPTB disease and drug resistance phenotypes hinder the generation of adequate epidemiological transmission chains and timely successful treatments. Therefore, this work aims to elucidate relationships between strain genotypes at the lineage/sublineage level and clinical disease phenotypes as well as antibiotic resistance. We retrieved the raw read datasets from the genomes of all the $M$. tuberculosis strains causing EPTB worldwide and deposited in databases. The raw datasets of the 
119

120

121

122

123

124

125

126

127

128

129

130

131

132

133

134

135

136

137

138

139

140

141

142

143

144

145

146

147

148

149

150

151

152

153

154

155

156

157

158

same number of genomes of strains causing PTB isolated from the same countries of origin as EPTB strains were selected to avoid sources of possible biases related to previous studies originating from (i) a low number of analyzed strains, (ii) unequal PTB/EPTB strains analyzed, or (iii) differences in regional clinical TB phenotype incidences. The epidemiological and public health relevance of the identified relationships is discussed.

\section{Materials \& Methods}

\section{Data retrieval}

A total of 490 raw datasets of genome sequence reads were retrieved, which corresponded to 245 M. tuberculosis strains causing PTB and 245 strains causing EPTB (Supplemental Table 1). We retrieved all available genomes of EPTB strains deposited in the NCBI-SRA database. Such genomes correspond to clinical cases for which the major site of infection reported in databases was not pulmonary or miliary and an additional infection site either was not specified or was specified but not as pulmonary or miliary (Click et al., 2012). The first criterion for the genome selection of the PTB strains was the country of the isolation of the EPTB strains for which the genomes were available. The objective of such a criterion was to analyze the genomes of $M$. tuberculosis phenotypes from similar clinical and human population backgrounds. The second criterion to select PTB strains was a negative HIV status reported in the associated metadata. In fact, this second criterion was applied for both PTB and EPTB genomes. Additionally, due to the large number of available genomes of PTB strains from different countries, it was possible to discard other comorbidities. All these criteria were used to avoid possible biases generated by population-genotype associations and HIV, or other comorbidities.

The sequence quality of the FASTQ reads was checked using FASTQC (http://www.bioinformatics.babraham.ac.uk/projects/fastqc) and subsequently filtered to a Phred score of 30 using TrimGalore (http://www.bioinformatics.babraham.ac.uk/projects/trim_galore/).

\section{In silico typing}

In silico spoligotyping was performed using SpoTyping program version 2.0 (Xia, Teo \& Ong, 2016) for next-generation sequencing reads with default parameters. To determine lineage, the TB INSIGHT (http://tbinsight.cs.rpi.edu) server was used based on the identified spoligotypes.

\section{Phylogenetic reconstruction}

To find SNPs for phylogenetic reconstruction (Homolka et al., 2012; Coll et al., 2014; Merker et al., 2015), the sequencing reads of the studied strains were aligned to the reference strain of $M$. tuberculosis H37Rv (accession no. NC_000962.3) using the MTBseq program version 1.0.3 (Kohl et al., 2018) with default values. A frequency of allelic variation greater or equal to $75 \%$ and with a phred value $>20$ was used. Strains where the percentage of reads mapped against the reference genome was less than 80 were excluded due to possible contamination. Also, strains 
159

160

161

162

163

164

165

166

167

168

169

170

171

172

173

174

175

176

177

178

179

180

181

182

183

184

185

186

187

188

189

190

191

192

193

194

195

196

197

198

with a median depth coverage $>30 x$ were removed. The minimum coverage depth to support a SNP was 8x. For lineage and sublineage assignment, variant positions belonging to repeated regions and resistance genes were excluded. Of the remaining variant positions, those where data quality is below thresholds in $>5 \%$ of samples were discarded (Jajou et al, 2019). The sublineage phylogeny was rooted using a Mycobacterium microti strain (SRR2667442). Phylogenetic inferences were conducted with the maximum likelihood (ML) criterion using the IQ-TREE package (Nguyen et al., 2015) with a general time-reversible (GTR) model of nucleotide substitution and a gamma model of rate heterogeneity. Phylogenetic trees were constructed based on 1,000 bootstrap replicates, and their visualization was performed using iTOL (Letunic \& Bork, 2016).

\section{In silico determination of drug resistance}

Raw FASTQ sequencing files were uploaded to TB-Profiler version 3.0.4 (Coll et al., 2015), a tool to determine in silico drug resistance. TB-Profiler can determine genotypic drug resistance by aligning raw sequences against the reference genome $M$. tuberculosis H37Rv in order to identify mutations (1541 SNPs and indels) previously associated with phenotypic drug-resistance from a curated database. This package also determines the M. tuberculosis lineage based on a 90SNP barcode. The TB-Profiler-predicted resistance mutations were validated using the results of MTBseq, which reports a list of mutations in genes associated with antimicrobial resistance for every processed strain.

\section{Data analysis}

Data entry and statistical analyses were performed in SPSS version 16 (SPSS Inc., Illinois, USA). Univariate analyses were performed using two-tailed Fisher's exact test to estimate the association between each variable (M. tuberculosis lineage, sublineage or genotypic resistance) and extrapulmonary tuberculosis relative to pulmonary tuberculosis. Additionally, each variable was compared to every anatomical site of TB disease (central nervous system, bones and joints, lymph nodes, and the genitourinary system). Odds ratios with $95 \%$ confidence intervals were considered an effect size of the association. To confirm that the association was not an artifact of demographic differences between the geographic regions, a logistic regression model was performed in which the EPTB group was included as a dependent variable. The variables geographic region (the country of isolation), sublineage and genotypic resistance were included in the model, and adjusted ORs with $95 \%$ confidence intervals were calculated.

\section{Results}

\section{Anatomical site of infection of strains selected for comparative genomics analysis}

A total of 490 raw genome datasets of $M$. tuberculosis strains isolated from seven different countries from individuals with a negative HIV infection status (Supplemental Table 1) were genotyped. The selected strains included those from countries where specific lineages 
199

200

201

202

203

204

205

206

207

208

209

210

211

212

213

214

215

216

217

218

219

220

221

222

223

224

225

226

227

228

229

230

231

232

233

234

235

236

237

238

predominate according to the SITVIT website (http://www.pasteurguadeloupe.fr:8081/SITVIT_ONLINE/), such as Thailand and Indonesia for the East Asian lineage and India for the East African-Indian lineage. Ninety percent of the selected strains were isolated from Indonesia, Thailand, and Russia. This high percentage is since most of the EPTB strains deposited in the NCBI-SRA database were isolated from these countries. Unfortunately, EPTB strains from African and American countries were not included in the conducted analysis because of the lack of genomes in the NCBI-SRA database until the completion of this study.

The EPTB phenotype strains were clustered into five major groups according to the anatomical site of infection, with a predominance of the central nervous system (72.24\%). Other anatomical sites of the disease were bones and joints (17.14\%), lymph nodes $(4.49 \%)$, and the genitourinary system $(2.86 \%)$; the remaining $3.27 \%$ were from sites specified as having only EPTB strains in databases.

\section{Distribution and association of lineages and genotypes with clinical phenotype}

The mean coverage obtained for the analyzed strain set was 109.82 , whereas the mean percentage of mapped reads was 98.28 , resulting in good genome coverage. The strains were assigned to a major $M$. tuberculosis genetic lineage according to the spoligotype using the TB lineage search option of the TB insight web server. These results correlated with the clustering pattern generated by the SNP-based phylogeny. The phylogenetic analysis of M. tuberculosis strains from PTB and EPTB phenotypes is shown in Figure 1. The predominant lineage of the analyzed strains was East Asian (54.28\%), followed by Euro-American (34.70\%), Indo-Oceanic (9.6\%), and East African-Indian (1.42\%). The EPTB case percentage differed among these phylogenetic lineages, with $15.51 \%$ for Indo-Oceanic, $48.98 \%$ for East Asian, $2.45 \%$ for East African-Indian, and $33.06 \%$ for Euro-American. The same variation in phylogenetic lineages was observed for strains from PTB cases, with $59.59 \%, 36.32 \%, 3.67 \%$, and $0.40 \%$ for the East Asian, Euro-American, Indo-Oceanic, and East African-Indian lineages, respectively. Fisher's exact test showed that PTB was significantly associated with East Asian lineage strains (OR 1.5; 95\% CI 1.1-2.2) and that EPTB was significantly associated with the Indo-Oceanic lineage strains (OR 4.8; 95\% CI 2.2-10.1) (Supplemental Table 2). After logistic regression analysis, the associations between L1 and EPTB (OR 4.4; 95\% CI 1.9-10.0 P=0.000) and L2 and PTB (OR $1.4 ; 95 \%$ CI 1.1-1.9) strains was confirmed, thus eliminating any potential bias due to the isolation regions of M. tuberculosis strains (Supplemental Table 2).

We searched for a relationship between the phylogenetic lineages found in the analyzed strains and anatomical sites of infection, including lungs, lymph nodes, the genitourinary system, the central nervous system, and bones and joints. The results showed that strains belonging to the Indo-Oceanic lineage were significantly associated with central nervous system infection (OR 3.9; CI 95\% 2.1-7.4) (Supplemental Table 3), whereas those of the East Asian lineage were significantly associated with bone and joint infection (OR 1.5; 95\% CI 1.3-1.9) and with PTB strains, as previously described. Further division of identified lineages was conducted using SNP analysis, distinguishing 27 unique sublineages, with the predominant sublineages being 2.2.1 
$239(\mathrm{n}=218$ strains $), 2.2 .1 .1(\mathrm{n}=25), 4.1 .2 .1(\mathrm{n}=18), 4.3 .1(\mathrm{n}=25), 4.8(\mathrm{n}=51)$, and 1.2.1 $(\mathrm{n}=22)$. As

240 expected, statistical analysis showed a significant relationship of some of these sublineages with

241 major infection sites. In this way, sublineage 1.1.1 was associated with central nervous system

242 infection (OR 2.8; 95\% CI 1.0-7.7), and the same relationship was observed for sublineages

243 1.2.1 (OR 6.7; 95\% CI 2.0-22.1) and 4.1.2.1 (OR 6.3; 95\% CI 1.9-21.1). On the other hand,

244 sublineages 4.3.1 (OR 2.9; 95\% CI 1.2-7.7), 4.3.3 (OR 1.8; 95\% CI 1.4-2.2), and 4.5 (OR 3.8;

$24595 \%$ CI 1.0-13.8) were associated with the pulmonary phenotype.

246 A deeper whole-genome SNP-based classification to discriminate the proto-Beijing from

247

248

249

250

251

252

253

254

255

256

257

258

259

260

261

262

263

264

265

266

267

268

269

270

271

272

273

274

275

276

277

278

the Beijing strains allowed us to identify the outbreak sublineages of Asia Ancestral 1, Asia Ancestral 3, Asian African 2, Asian African 2/RD142, Asian African 3, Pacific RD150, Central Asia, and Europe/Russia B0/W148 and a group of modern unclassified strains using informative genetic markers found in the genomes of the studied strains (Figure 2). Of all these genotypes, the Asian African 2 sublineage (OR 2.3; 95\% CI 1.1-5.2) and Europe/Russia B0/W148 outbreak sublineage (OR 2.7; 95\% CI 1.3-5.4) were significantly associated with PTB. In the same way, the Ancestral Beijing sublineages (Asia Ancestral 1 and Asia Ancestral 3) (OR 2.4; 95\% CI 1.24.8) and Central Asia subgroup (OR 8.2; 95\% CI 2.9-22.9) were significantly associated with EPTB.

The most frequent spoligotype among L2 strains (85.1\%) was SIT1 (Supplemental Table 4). SIT 53 and SIT 19 were the most frequent spoligotypes for the L4 (22.9\%) and L1 (27.6\%) strains, respectively. For the L3 strains, $42 \%$ had an unclassified SIT spoligotype (703775740003771). Spoligotyping allowed the identification of twelve major genotypic families, including Beijing (53.7\%), Central Asia (CAS) (1.4\%), EAI (9.8\%), Haarlem (4.4\%), Latin American-Mediterranean (LAM) (7.5\%), S (0.2\%), T (19.8\%), X (0.4\%) and Family33-36 (2\%). Interestingly, $13.26 \%$ of the strains corresponded to spoligotypes not previously reported in the SITVIT database (Supplemental Table 4). A significant association was found between the EAI2 family (OR 6.7; 95\% CI 2.0-22.1) and EPTB, of which 13 strains caused tuberculous meningitis and two of them led to lymph node infection.

\section{Mutations associated with drug-resistant TB}

A total of 180 previously reported mutations distributed in 18 genes known to confer resistance to first- and second-line drugs for TB treatment were identified (Supplemental Table 5).

Isoniazid was the antibiotic with the highest predicted resistance in $38.77 \%$ of the studied strains, followed by rifampicin in $33.46 \%$, streptomycin in $31.02 \%$ and ethambutol in $27.14 \%$.

The most common mutation associated with isoniazid resistance was $k a t G \operatorname{Ser} 315 \mathrm{Thr}$, which was found in $82.63 \%$ of the genotypic resistant strains. rpoB Ser450Leu was found in $52.43 \%$ of the rifampicin-resistant strains, $r p s L$ Lys $43 \mathrm{Arg}$ in $72.36 \%$ of streptomycin-resistant strains and embB Met306Val in $33.83 \%$ of ethambutol-resistant strains.

Extrapulmonary strains showed greater diversity of mutations but appeared less frequently than pulmonary strains. Mutations gyrA (Ala90Val) and thyX (Glu40Gly) were found only in pulmonary strains. On the other hand, mutations in the rpoB (Leu430Pro), rrs (907

Peer) reviewing PDF | (2021:05:60932:2:0:NEW 10 Aug 2021) 
$279 \mathrm{~A}>\mathrm{C}), r p s L$ (Lys88Thr), embA (16C $>\mathrm{G})$, embB (Ala356Val, Ser347Ile), ethA (Gln269*, 280 Gly385Asp), katG (589 insGG), and pncA (Ala3Glu, Cys138Arg, Cys72Arg, Gln10Pro, 281 His51 Asp, Gly97Asp, Pro54Leu, Thr135Pro) genes were found only in extrapulmonary strains. 282 There were no statistically significant differences among these variations with the tuberculosis 283 clinical phenotype.

284 Differences between the distribution of drug-resistant (26.9\% PTB, 15.9\% EPTB) and 285 drug-sensitive (23.1\% PTB, 34.1\% EPTB) strains were observed in the EPTB and PTB groups. 286 Four resistance profiles were determined: sensitive, drug resistance (DR), multidrug resistance 287 MDR, and extensive drug resistance (XDR). The frequency for each EPTB/PTB group is shown 288 in Supplemental Table 2. The bone and joint group presented the highest number of strains with 289 antibiotic resistance: $76.1 \%$ were resistant to isoniazid, $73.8 \%$ to streptomycin, $66.6 \%$ to 290 rifampicin, $54.7 \%$ to ethambutol, $42.8 \%$ to pyrazinamide and ethionamide, $14.2 \%$ to

291

292

293

294

295

296

297

298

299

300

301

302

303

304

305

306

307

308

309

310

311

312

313

314

315

316

317

318 fluoroquinolones and 9.5\% to aminoglycosides and paraaminosalicylic acid (Supplemental Table 6). A total of $13.5 \%$ of central nervous system strains showed resistance to isoniazid, and $54.4 \%$ of lymph node strains were resistant to rifampicin. Sensitive strains were statistically associated with EPTB (OR: 1.7; 95\% CI 1.3-2.3), and extensive drug resistance (XDR; OR: 6.0; 95\% IC 2.5-14.4) was more strongly associated with PTB strains than with EPTB strains. After logistic regression analysis including the geographic region of isolation, XDR strains were still associated with pulmonary rather than extrapulmonary disease (OR: 6.0; 95\% IC 2.3-14.4 $\mathrm{P}=0.000$ ). We also observed a significant association of Lineage 3 strains with MDR (OR: 3.8 ; 95\% CI 1.1-13.6) and between the L2 lineage and XDR resistance (OR: 8.1; 5.4-8.6).

\section{Discussion}

In this work, we used both raw genome datasets and whole-genome SNP-based phylogeny to genotype the same number of PTB and EPTB M. tuberculosis strains. All the strains were included in one of the six lineages defined by Coll (Coll et al., 2014). When seeking lineageclinical phenotype relationships, we found that the East Asian 'modern' M. tuberculosis lineages were associated with PTB, whereas the Indo-Oceanic so-called an 'ancient lineage' were associated with EPTB. It is known that strains from 'modern' lineages induced a slighter inflammatory response than those from 'ancient' lineages, which has been related to a selective advantage of strains from 'modern' lineages, resulting in impaired bacterial control by the host, faster disease progression, and enhanced transmission (Portevin et al., 2011). These clinical differences contribute to explaining the association of the East Asian lineage with the PTB phenotype, supporting the hypothesis that since this is the disease contagious phenotype, patients infected with strains of such a lineage are prone to develop pulmonary disease at a higher frequency than patients infected with strains from other genotypes, which is consistent with the increased transmissibility of strains from this lineage (Thwaites et al., 2018).

It has been proposed that strains belonging to the Indo-Oceanic lineage are 'less virulent' than those from other lineages and cause a specific exacerbated inflammatory response 
319 (Chakraborty et al., 2018), which might be attributed to the presence of unique cell envelope 320 lipids in this lineage, such as phenol phthiocerol dimycocerosate (Krishnan et al., 2011).

321 Nevertheless, it is still unknown whether these differences in mycobacterial cell envelope lipid 322 composition can explain lineage-related phenotypic differences such as the EPTB phenotype.

323 Interestingly, the percentage of EPTB cases associated with the East African-Indian lineage was 324 as high as $85 \%$, but this finding was not statistically significant due to the small number of EPTB

325

326

327

328

329

330

331

332

333

334

335

336

337

338

339

340

341

342

343

344

345

346

347

348

349

350

351

352

353

354

355

356

357

358 raw genomes $(\mathrm{n}=7)$ from this lineage available in databases. In this regard, the present work reveals database gaps relevant for its public health and epidemiological implications, i.e., the need to include more East African-Indian lineage genomes to clarify its relationships with EPTB.

In the search for a detailed relationship between phylogenetic lineages and the anatomical site of infection, strains belonging to the East Asian lineage were associated with the infection of both bones and joints and PTB. The East Asian lineage comprises two major clades or sublineages, designated proto-Beijing (2.1) and Beijing (2.2) (Ajawatanawong et al., 2019). Sublineage 2.2, or the Beijing family, as defined by spoligotyping, is composed of several sublineages broadly categorized into the ancestral and modern Beijing strains (Mokrousov et al., 2005). A subtle SNP-based classification was recently proposed that allows the discrimination of proto-Beijing from Beijing strains (Shitikov et al., 2017). Such classification divides the Beijing group into the ancestral Beijing clade and the modern Beijing clade, which comprises two groups, one including three strains (Asia Ancestral 1, Asia Ancestral 2, Asia Ancestral 3) and the other including seven strains (Asian African 1, Asian African 2, Asian African 2/RD142, Asian African 3, Pacific RD150, Europe/Russia B0/W148 outbreak and Central Asia). This classification allows us to associate the Asian African 2 sublineage and Europe/Russia B0/W148 outbreak sublineage with PTB, as well as the ancestral Beijing sublineages and Central Asia subgroup with EPTB. Differences in the pathogenicity of Beijing sublineages have been previously reported (Feng et al., 2008), and sublineage specific patterns of induced cytokine production by macrophages have also been observed (Sarkar et al., 2012). In this regard, a macrophage infection model revealed that ancestral Beijing strains induce a higher production of the proinflammatory cytokines TNF- $\alpha$ and IL-6 than the modern Beijing sublineage (Chen et al., 2014). High IFN- $\gamma$ expression and cytokine production have also been reported in peripheral blood mononuclear cells of ancestral Beijing strains (Faksri et al., 2014). Such results suggest that the ancestral Beijing strains are as highly immunogenic (Kato-Maeda et al., 2012) as Lineage 1 strains (EAI) (Rakotosamimanana et al., 2010). Interestingly, these two sublineages (EAI and ancestral Beijing) were associated with the EPTB phenotype in this study. In contrast, the other sublineage associated with EPTB in this work, 4.1.2.1, has been reported to induce cytokine levels similar to those of H37Rv (Haarlem family strains), which are associated with a low immune response (Wang et al., 2010). This supports the idea that strains of different sublineages vary by many phenotypes, such as the tendency to develop drug resistance, virulence levels, and immune response, which influence disease severity and clinical presentation.

Interestingly, sublineages 4.1.2.1 and ancestral Beijing, belonging to Lineages 4 and 2, respectively, show a high prevalence worldwide, representing more than $50 \%$ of the strains in 
359

360

361

362

363

364

365

366

367

368

369

370

371

372

373

374

375

376

377

378

379

380

381

382

383

384

385

386

387

388

389

390

391

392

393

394

395

396

397

398

certain areas and/or subpopulations (Ajawatanawong et al., 2019). In contrast, Lineage 1 (EAI) strains are commonly reported in countries around the Indian Ocean and are one of the most geographically restricted families. However, EAI strains have been reported in lower percentages in countries such as the Netherlands, Australia, the USA, Sweden, Saudi Arabia, Tunisia, Taiwan, Panama, and Mexico. The East Asian India 2 spoligotype of Lineage 1 corresponds to the Nonthaburi (EAI2-Nonthaburi) genotype and the Manila (EAI2-Manila) genotype (Couvin, Reynaud \& Rastogi, 2019). Recently, Coker (Coker et al. 2016) reported a polymorphism in the genome of Nonthaburi strains from three patients with tuberculous meningitis, consisting of a $500 \mathrm{bp}$ deletion covering ppe50 that was not present in the reference strain M. tuberculosis H37Rv. They reported three mutations (T28910C, C1180580T and, C152178T) until now found only in these meningeal Nonthaburi strains. These mutations could represent part of the genetic background that could be shared by strains that cause EPTB and that also belong to the EAI, 4.1.2.1, and ancestral Beijing sublineages. Nonetheless, further investigation is required to determine whether these mutations are shared, which could be the functional consequences and the probable relation of such polymorphisms with TB disease phenotype.

Regarding the genotype relationship with antibiotic resistance, to the best of our knowledge, a significant association of Lineage 3 with drug resistance found here has not been previously reported. This result might be useful to optimize TB treatment in geographical areas where this lineage is frequent. Studies from Asia, Europe and Africa have shown varying associations between drug resistance and MTB lineages (Coscolla \& Gagneux, 2010; Singh et al., 2015); however, as was found here, Beijing strains have been associated with MDR and XDR in several cases (Rodríguez-Castillo et al., 2017). However, it must be highlighted that the lack of experimental drug resistance assays is a limitation of this study. The bioinformatic approach to determine the drug resistance of the studied isolates is based on software packages that use different sets of mutations in their analysis. This generates a variation in the drugresistant genotypes obtained with the different bioinformatic tools with the consequent risk of over- or underestimating the true drug-resistant behavior of the analyzed strains. The statistical inferences that depend on this determination are also subject to such biases as the association of drug resistance with affected organs or mycobacterial lineages. Thus, it is strongly recommended to perform the experimental determination of the drug resistance phenotype of the strains in which the genome will be deposited in public databases.

The advantages of the present study regarding previous works seeking relationships of the $M$. tuberculosis genotype with clinical phenotype include the use of a significantly higher number of strains, an equal number of EPTB and PTB strains, a clear assignment of the strains considered to be associated with EPTB, and the exclusion of the strains from HIV patients, with the latter being a controlled variable, a caution not commonly considered in similar works. However, other possible confounders or known risk factors for EPTB could not be considered in the analysis conducted here, partly due to the lack of additional metadata of clinical information in the database where the genomes of the strains were retrieved. This lack of metadata is not a source of bias in the relationships found here because it has been shown that there is an 
399

400

401

402

403

404

405

406

407

408

409

410

411

412

413

414

415

416

417

418

419

420

421

422

423

424

425

426

427

428

429

430

431

432

433

434

435

436

437

438

independent association between lineages and EPTB rather than ethnic factors after a stratified analysis (Click et al., 2012). Additionally, it must be noted that the inclusion of genomes previously published that can be generated for different purposes can generate a bias by sampling protocols to seek specific pathogen genotypes or tuberculosis phenotypes. This was a not controlled variable of this study.

These results support the assertion that the relationship between sublineages and clinical disease phenotypes is not attributable to regional-specific factors; therefore, if this association exists, it does not depend only on unknown clinical factors. However, the contribution of the genetic background of the host to the occurrence of the different clinical phenotypes of tuberculosis should not be ruled out. Several polymorphisms associated with EPTB have been previously reported in specific human populations (Fox et al., 2014). Therefore, associations found here must be further tested with additional epidemiological data to clarify the possible relationship between the intrinsic pathogen sublineage characteristics and host factors as genetic background for the establishment of the infection in a particular organ.

\section{Conclusions}

Overall, the obtained results suggest that intralineage diversity could drive differences in the immune response that trigger the different clinical phenotypes of tuberculosis. The immune response caused by ancient lineage (L1) and ancestral Beijing strains could be similar, eliciting a high inflammatory response. Our results highlight the need both to analyze the genomic background shared by these strains and to perform in vitro/in vivo studies that help to elucidate the mechanism by which they could disseminate through the body, causing extrapulmonary disease. We demonstrated that the lack of consistency regarding clinical phenotype-strain genotype associations in the results obtained by previous studies is partially due to the use of inadequate phylogenetic classification tools, which do not allow discrimination between sublineages. Additionally, the present results were not biased by the predominance of a specific lineage/sublineage in a specific human population because we included all the EPTB strains available in databases and an evenly representative sample of PTB strains. Biases originating from unknown sampling procedures of the strains for which genomes are available in databases and due to unknown phenotypic drug resistance are the main limitations of the present study.

\section{Acknowledgements}

We thank CONACYT-Mexico for scholarship No. 701889 granted to A.M.N.P., and Coordinación de la Investigación Científica UMSNH research program; We appreciate the criticism of Dr. Sebastien Gagneux and an anonymous reviewer that contributed to significantly improve the manuscript.

\section{References}


439

440

441

442

443

444

445

446

447

448

449

450

451

452

453

454

455

456

457

458

459

460

461

462

463

464

465

466

467

468

469

470

471

472

473

474

475

476

477

478

479

480

481

482

Ajawatanawong P, Yanai H, Smittipat N, Disratthakit A, Yamada N, Miyahara R, Nedsuwan S, Imasanguan W, Kantipong P, Chaiyasirinroje B, Wongyai J, Plitphonganphim S, Tantivitayakul P, Phelan J, Parkhill J, Clark TG, Hibberd ML, Ruangchai W, Palittapongarnpim P, Juthayothin T, Thawornwattana Y, Viratyosin W, Tongsima S, Mahasirimongkol S, Tokunaga K, Palittapongarnpim P. 2019. A novel Ancestral Beijing sublineage of Mycobacterium tuberculosis suggests the transition site to Modern Beijing sublineages. Scientific Reports 9: 1-12 DOI:10.1038/s41598-019-50078-3

Chakraborty P, Kulkarni S, Rajan R, Sainis K. 2018. Mycobacterium tuberculosis strains from ancient and modern lineages induce distinct patterns of immune responses. Journal of Infection in Developing Countries 11: 904-911 DOI:10.3855/jidc.8596

Chen YY, Chang JR, Huang WF, Hsu SC, Kuo SC, Sun JR, Dou HY. 2014. The pattern of cytokine production in vitro induced by ancient and modern Beijing Mycobacterium tuberculosis strains. PLOS ONE 9: 1-7 DOI:10.1371/journal.pone.0094296

Click ES, Moonan PK, Winston CA, Cowan LS, Oeltmann JE. 2012. Relationship between Mycobacterium tuberculosis phylogenetic lineage and clinical site of tuberculosis. Clinical Infectious Disease 54: 211-219 DOI:10.1093/cid/cir788

Coker OO, Chaiprasert A, Ngamphiw C, Tongsima S, Regmi SM, Clark TG, Ong RT, Teo YY, Prammananan T, Palittapongarnpim P. 2016. Genetic signatures of Mycobacterium tuberculosis Nonthaburi genotype revealed by whole genome analysis of strains from tuberculous meningitis patients in Thailand. PeerJ 4: 1-20 DOI:10.7717/peerj.1905

Coll F, McNerney R, Guerra-Assunção JA, Glynn JR, Perdigão J, Viveiros M, Portugal I, Pain A, Martin N, Clark TG. 2014. A robust SNP barcode for typing Mycobacterium tuberculosis complex strains. Nature communications 5: 1-5 DOI:10.1038/ncomms5812

Coll F, McNerney R, Preston MD, Guerra-Assunção JA, Warry A, Hill-Cawthorne G, Mallard K, Nair M, Miranda A, Alves A, Perdigão J, Viveiros M, Portugal I, Hasan Z, Hasan R, Glynn JR, Martin N, Pain A, Clark TG. 2015. Rapid determination of anti-tuberculosis drug resistance from whole-genome sequences. Genome Medicine 7: 51-61 DOI:10.1186/s13073-015-0164-0

Coscolla M, Gagneux S, Menardo F, Loiseau C, Ruiz-Rodriguez P, Borrell S, Otchere ID, Asante-Poku A, Asare P, Sánchez-Busó L, Gehre F, Sanoussi CN, Antonio M, Affolabi D, Fyfe J, Beckert P, Niemann S, Alabi AS, Grobusch MP, Kobbe R, Parkhill J, Beisel C, Fenner L, Böttger EC, Meehan CJ, Harris SR, de Jong BC, Yeboah-Manu D, Brites D. 2021. Phylogenomics of Mycobacterium africanum reveals a new lineage and a complex evolutionary history. Microbial Genomics 7:1-14 DOI:10.1099/mgen.0.000477

Coscolla M, Gagneux S. 2010. Does M. tuberculosis genomic diversity explain disease diversity?. Drug Discovery Today Disease Mechanisms 7: 1-26

DOI:10.1016/j.ddmec.2010.09.004

Coscolla M, Gagneux S. 2014. Consequences of genomic diversity in Mycobacterium tuberculosis. Seminars in Immunology 26: 431-44 DOI:10.1016/j.smim.2014.09.012

Couvin D, Reynaud Y, Rastogi N. 2019. Two tales: worldwide distribution of Central Asian (CAS) versus ancestral East-African Indian (EAI) lineages of Mycobacterium tuberculosis underlines a remarkable cleavage for phylogeographical, epidemiological and demographical characteristics. PLoS ONE 14: 1-20

doi.org/10.1371/journal.pone.0219706

Peer] reviewing PDF | (2021:05:60932:2:0:NEW 10 Aug 2021) 
483

484

485

486

487

488

489

490

491

492

493

494

495

496

497

498

499

500

501

502

503

504

505

506

507

508

509

510

511

512

513

514

515

516

517

518

519

520

521

522

523

524

525

526

527

528
Dale JW, Bothamley GH, Drobniewski F, Gillespie SH, McHugh TD, Pitman R. 2005. Origins and properties of Mycobacterium tuberculosis strains in London. Journal of Medical Microbiology 54: 575-582 DOI:10.1099/jmm.0.45959-0

Faksri K, Chaiprasert A, Pardieu C, Casali N, Palaga T, Prammananan T, Palittapongarnpim P, Prayoonwiwat N, Drobniewski F. 2014. Heterogeneity of phenotypic characteristics of the modern and ancestral Beijing strains of Mycobacterium tuberculosis. Asian Pacific Journal of Allergy and Immunology 32: 124-132 DOI:10.12932/AP0361.32.2.2013

Faksri K, Xia E, Ong RT, Tan JH, Nonghanphithak D, Makhao N, Thamnongdee N, Thanormchat A, Phurattanakornkul A, Rattanarangsee S, Ratanajaraya C, Suriyaphol P, Prammananan T, Teo YY, Chaiprasert A. 2018. Comparative whole-genome sequence analysis of Mycobacterium tuberculosis isolated from tuberculous meningitis and pulmonary tuberculosis patients. Scientific Reports 8: 1-10 DOI:10.1038/s41598-01823337-y

Feng JY, Su WJ, Tsai CC, Chang SC. 2008. Clinical impact of Mycobacterium tuberculosis WBeijing genotype strain infection on aged patients in Taiwan. Journal of Clinical Microbiology 46: 3127-3129 DOI:10.1128/JCM.01132-08

Firdessa R, Berg S, Hailu E, Schelling E, Gumi B, Erenso G, Gadisa E, Kiros T, Habtamu M, Hussein J, Zinsstag J, Robertson BD, Ameni G, Lohan AJ, Loftus B, Comas I, Gagneux S, Tschopp R, Yamuah L, Hewinson G, Gordon SV, Young DB, Aseffa A. 2013. Mycobacterial lineages causing pulmonary and extrapulmonary tuberculosis, Ethiopia. Emerging Infectious Disease 19: 460-463 DOI:10.3201/eid1903.120256.

Floyd K, Glaziou P, Zumla A, Raviglione M. 2018. The global tuberculosis epidemic progress in patient care, prevention and control efforts in year 3 of the End TB Era. Lancet Respiratory Medicine 6: 299-314 DOI:10.1016/S2213-2600(18)30057-2

Fox GJ, Sy DN, Nhung NV, Yu B, Ellis MK, Van Hung N, Cuong NK, Thi Lien L, Marks GB, Saunders BM, Britton WJ. 2014. Polymorphisms of SP110 are associated with both pulmonary and extra-pulmonary tuberculosis among the Vietnamese. PLoS One. 9: 1-9 DOI:10.1371/journal.pone.0099496.

Gagneux S. 2018. Ecology and evolution of Mycobacterium tuberculosis. Nature Reviews Mycrobiology 16: 202-213 DOI: 10.1038/nrmicro.2018.8

Golden MP, Vikram HR. 2005. Extrapulmonary tuberculosis: an overview. American Family Physician 72: 1761-1768.

Homolka S, Projahn M, Feuerriegel S, Ubben T, Diel R, Nübel U, Niemann S. 2012. High resolution discrimination of clinical Mycobacterium tuberculosis complex strains based on single nucleotide polymorphisms. PLOS ONE 7:1-11

DOI:10.1371/journal.pone.0039855

Jajou R, Kohl TA, Walker T, Norman A, Cirillo DM, Tagliani E, Niemann S, de Neeling A, Lillebaek T, Anthony RM, van Soolingen D. 2019. Towards standardisation: comparison of five whole genome sequencing (WGS) analysis pipelines for detection of epidemiologically linked tuberculosis cases. Eurosurveillance. 24: 1-10 DOI: 10.2807/1560-7917.ES.2019.24.50.1900130.

Kato-Maeda M, Nahid P. 2012. Mycobacterium tuberculosis lineage-what's in your lungs?. Clinical Infectious Disease 54: 220-224 DOI:10.1093/cid/cir795

Kato-Maeda M, Shanley CA, Ackart D, Jarlsberg LG, Shang S, Obregon-Henao A, Harton M, Basaraba RJ, Henao-Tamayo M, Barrozo JC, Rose J, Kawamura LM, Coscolla M, Fofanov VY, Koshinsky H, Gagneux S, Hopewell PC, Ordway DJ, Orme IM. 2012.

Peer] reviewing PDF | (2021:05:60932:2:0:NEW 10 Aug 2021) 
529

530

531

532

533

534

535

536

537

538

539

540

541

542

543

544

545

546

547

548

549

550

551

552

553

554

555

556

557

558

559

560

561

562

563

564

565

566

567

568

569

570

571

572

573

Beijing sublineages of Mycobacterium tuberculosis differ in pathogenicity in the guinea pig. Clinical Vaccine Immunology 19: 1227-1237 DOI:10.1128/CVI.00250-12

Kohl TA, Utpatel C, Schleusener V, De Filippo MR, Beckert P, Cirillo DM, Niemann S. 2018. MTBseq: A comprehensive pipeline for whole genome sequence analysis of Mycobacterium tuberculosis complex strains. PeerJ 11: 1-13 DOI:10.7717/peerj.5895

Krishnan N, Malaga W, Constant P, Caws M, Tran TH, Salmons J, Nguyen TN, Nguyen DB, Daffé M, Young DB, Robertson BD, Guilhot C, Thwaites GE. 2011. Mycobacterium tuberculosis lineage influences innate immune response and virulence and is associated with distinct cell envelope lipid profiles. PLOS ONE 6: 1-6 DOI: 10.1371/journal.pone.0023870.

Letunic I, Bork P. 2016. Interactive tree of life (iTOL) v3: an online tool for the display and annotation of phylogenetic and other trees. Nucleic Acids Research 44: W242-245 DOI:10.1093/nar/gkw290

Malik AN, Godfrey-Faussett P. 2005. Effects of genetic variability of Mycobacterium tuberculosis strains on the presentation of disease. Lancet Infectious Disease 5: 174-83 DOI: 10.1016/S1473-3099(05)01310-1

McHenry ML, Bartlett J, Igo RP, Wampande EM, Benchek P, Mayanja-Kizza H, Benchek P, Mayanja-Kizza H, Fluegge K, Hall NB, Gagneux S, Tishkoff SA, Wejse C, Sirugo G, Boom WH, Joloba M, Williams SM, Stein CM. 2020. Interaction between host genes and Mycobacterium tuberculosis lineage can affect tuberculosis severity: Evidence for coevolution?. PLoS genetics, 16: 1-18. DOI: 10.1371/journal.pgen.1008728.

Merker M, Blin C, Mona S, Duforet-Frebourg N, Lecher S, Willery E, Blum MG, Rüsch-Gerdes S, Mokrousov I, Aleksic E, Allix-Béguec C, Antierens A, Augustynowicz-Kopeć E, Ballif M, Barletta F, Beck HP, Barry CE 3rd, Bonnet M, Borroni E, Campos-Herrero I, Cirillo D, Cox H, Crowe S, Crudu V, Diel R, Drobniewski F, Fauville-Dufaux M, Gagneux S, Ghebremichael S, Hanekom M, Hoffner S, Jiao WW, Kalon S, Kohl TA, Kontsevaya I, Lillebæk T, Maeda S, Nikolayevskyy V, Rasmussen M, Rastogi N, Samper S, Sanchez-Padilla E, Savic B, Shamputa IC, Shen A, Sng LH, Stakenas P, Toit K, Varaine F, Vukovic D, Wahl C, Warren R, Supply P, Niemann S, Wirth T. 2015. Evolutionary history and global spread of the Mycobacterium tuberculosis Beijing lineage. Nature Genetics 47: 242-249 DOI:10.1038/ng.3195

Mokrousov I, Ly HM, Otten T, Lan NN, Vyshnevskyi B, Hoffner S, Narvskaya O. 2005. Origin and primary dispersal of the Mycobacterium tuberculosis Beijing genotype: clues from human phylogeography. Genome Research 15: 1357-1364 DOI:10.1101/gr.3840605

Ngabonziza JCS, Loiseau C, Marceau M, Jouet A, Menardo F, Tzfadia O, Antoine R, Niyigena EB, Mulders W, Fissette K, Diels M, Gaudin C, Duthoy S, Ssengooba W, André E, Kaswa MK, Habimana YM, Brites D, Affolabi D, Mazarati JB, de Jong BC, Rigouts L, Gagneux S, Meehan CJ, Supply P. 2020. A sister lineage of the Mycobacterium tuberculosis complex discovered in the African Great Lakes region. Nature communications 11: 1-11 DOI:10.1038/s41467-020-16626-6

Nguyen L. 2016. Antibiotic resistance mechanisms in M. tuberculosis: an update. Archives of Toxicology 90:1585-1604 DOI:10.1007/s00204-016-1727-6

Nguyen LT, Schmidt HA, von Haeseler A, Minh BQ. 2015. IQ-TREE: a fast and effective stochastic algorithm for estimating maximum-likelihood phylogenies. Molecular Biology and Evolution 32: 268-274 DOI:10.1093/molbev/msu300 
574 Portevin D, Gagneux S, Comas I, Young D. 2011. Human macrophage responses to clinical $575 \quad$ strains from the Mycobacterium tuberculosis complex discriminate between ancient and

576

577

578

579

580

581

582

583

584

585

586

587

588

589

590

591

592

593

594

595

596

597

598

599

600

601

602

603

604

605

606

607

608

609

610

611

612

613

614

615

616

617

618

619 modern lineages. PLoS Pathogens 7: 1-12 DOI:10.1371/journal.ppat.1001307

Rakotosamimanana N, Raharimanga V, Andriamandimby SF, Soares JL, Doherty TM, Ratsitorahina M, Ramarokoto H, Zumla A, Huggett J, Rook G, Richard V, Gicquel B, Rasolofo-Razanamparany V; VACSEL/VACSIS Study Group. 2010. Variation in gamma interferon responses to different infecting strains of Mycobacterium tuberculosis in acidfast bacillus smear-positive patients and household contacts in Antananarivo, Madagascar. Clinical Vaccine Immunology 17: 1094-1203 DOI:10.1128/CVI.00049-10

Rodríguez-Castillo JG, Pino C, Niño LF, Rozo JC, Llerena-Polo C, Parra-López CA, Tauch A, Murcia-Aranguren MI. 2017. Comparative genomic analysis of Mycobacterium tuberculosis Beijing-like strains revealed specific genetic variations associated with virulence and drug resistance. Infection Genetics and Evolution 54: 314-323 DOI:10.1016/j.meegid.2017.07.022

Sarkar R, Lenders L, Wilkinson KA, Wilkinson RJ, Nicol MP. 2012. Modern lineages of Mycobacterium tuberculosis exhibit lineage-specific patterns of growth and cytokine induction in human monocyte-derived macrophages. PLOS ONE 7: 1-8 DOI:10.1371/journal.pone.0043170

Shitikov E, Kolchenko S, Mokrousov I, Bespyatykh J, Ischenko D, Ilina E, Govorun V. 2017. Evolutionary pathway analysis and unified classification of East Asian lineage of Mycobacterium tuberculosis. Scientific Reports 7: 1-10 DOI:10.1038/s41598-017-100185

Singh J, Sankar MM, Kumar P, Couvin D, Rastogi N, Singh S, Indian TB Diagnostics Network. 2015. Genetic diversity and drug susceptibility profile of Mycobacterium tuberculosis isolated from different regions of India. Journal of Infectology 71: 207-219 DOI:10.1016/j.jinf.2015.04.028

Stucki D, Brites D, Jeljeli L, Coscolla M, Liu Q, Trauner A, Fenner L, Rutaihwa L, Borrell S, Luo T, Gao Q, Kato-Maeda M, Ballif M, Egger M, Macedo R, Mardassi H, Moreno M, Tudo Vilanova G, Fyfe J, Globan M, Thomas J, Jamieson F, Guthrie JL, Asante-Poku A, Yeboah-Manu D, Wampande E, Ssengooba W, Joloba M, Henry Boom W, Basu I, Bower J, Saraiva M, Vaconcellos SEG, Suffys P, Koch A, Wilkinson R, Gail-Bekker L, Malla B, Ley SD, Beck HP, de Jong BC, Toit K, Sanchez-Padilla E, Bonnet M, GilBrusola A, Frank M, Penlap Beng VN, Eisenach K, Alani I, Wangui Ndung'u P, Revathi G, Gehre F, Akter S, Ntoumi F, Stewart-Isherwood L, Ntinginya NE, Rachow A, Hoelscher M, Cirillo DM, Skenders G, Hoffner S, Bakonyte D, Stakenas P, Diel R, Crudu V, Moldovan O, Al-Hajoj S, Otero L, Barletta F, Jane Carter E, Diero L, Supply P, Comas I, Niemann S, Gagneux S. 2016. Mycobacterium tuberculosis lineage 4 comprises globally distributed and geographically restricted sublineages. Nature Genetics 48: 15351543 DOI:10.1038/ng.3704.

Svensson E, Millet J, Lindqvist A, Olsson M, Ridell M, Rastogi N; Western Sweden Tuberculosis Epidemiology Study Group. 2011. Impact of immigration on tuberculosis epidemiology in a low-incidence country. Clinical Microbiology and Infection 17: 881887 DOI:10.1111/j.1469-0691.2010.03358.x

Thwaites G, Caws M, Chau TT, D'Sa A, Lan NT, Huyen MN, Gagneux S, Anh PT, Tho DQ, Torok E, Nhu NT, Duyen NT, Duy PM, Richenberg J, Simmons C, Hien TT, Farrar J. 2018. Relationship between Mycobacterium tuberculosis genotype and the clinical

PeerJ reviewing PDF | (2021:05:60932:2:0:NEW 10 Aug 2021) 
620

621

622

623

624

625

626

627

628

629

630

631 phenotype of pulmonary and meningeal tuberculosis. Journal of Clinical Microbiology 46: 1363-1368 DOI:10.1128/JCM.02180-07

Wang C, Peyron P, Mestre O, Kaplan G, van Soolingen D, Gao Q, Gicquel B, Neyrolles O. 2010. Innate immune response to Mycobacterium tuberculosis Beijing and other genotypes. PLoS ONE 5: 1-8 DOI:10.1371/journal.pone.0013594

Wang XH, Ma AG, Han XX, Gu XM, Fu LP, Li PG, Li FY, Wang QZ, Liang H, Katar A, Wang LJ. 2015. Correlations between drug resistance of Beijing/W lineage clinical strains of Mycobacterium tuberculosis and sublineages: a 2009-2013 prospective study in Xinjiang province, China. Medical Science Monitor 21: 1313-1318 DOI:10.12659/MSM.892951

Xia E, Teo YY, Ong RT. 2016. SpoTyping: Fast and accurate in silico Mycobacterium spoligotyping from sequence reads. Genome Medicine 8: 1-9 DOI:10.1186/s13073-016$0270-7$ 


\section{Figure 1}

Phylogenetic analysis of $M$. tuberculosis strains from PTB and EPTB phenotypes of the disease. Sensitive, does not present genotypic resistance; Drug-resistant, resistant to at least one antibiotic; MDR, resistant to at least isoniazid and rifampicin

Sensitive, does not present genotypic resistance; Drug-resistant, resistant to at least one antibiotic; MDR, resistant to at least isoniazid and rifampicin; XDR, resistant to isoniazid and rifampicin plus any fluoroquinolone and at least one of three injectable second-line drugs. The phylogenetic tree was inferred using the maximum likelihood $(\mathrm{ML})$ criterion with a general time-reversible model of nucleotide substitution and a gamma model of rate heterogeneity. Yellow highlighted letters indicate EPTB strains. Support values correspond to bootstrap values. The topology was rooted with a Mycobacterium microti strain.

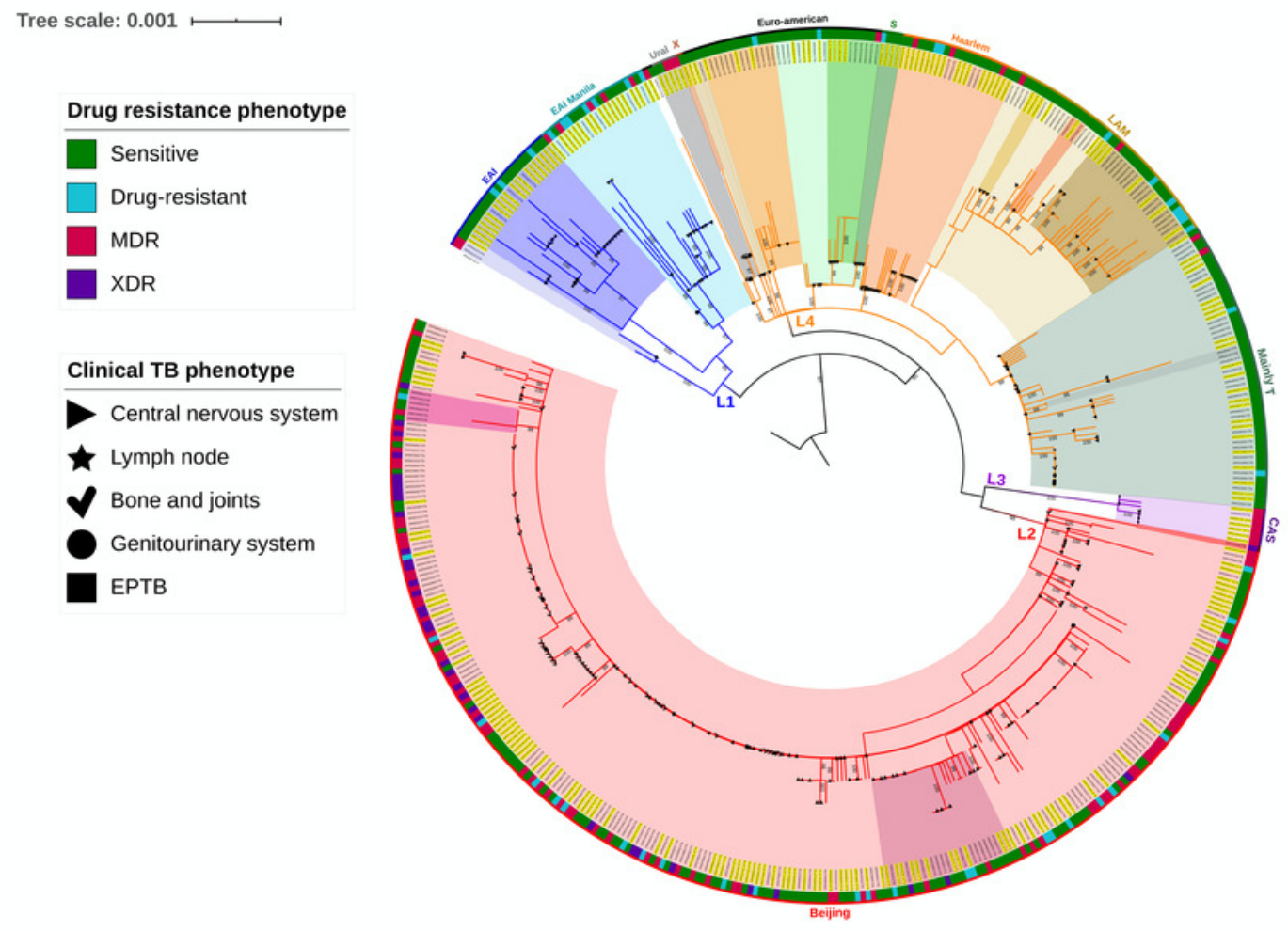




\section{Figure 2}

Phylogeny of 263 Mycobacterium tuberculosis L2 strains. The phylogeny was constructed by the maximum likelihood (ML) criterion. Classification of strains into sublineages and informative genetic markers are shown. Support values correspond to bootstrap va

The phylogeny was constructed by the maximum likelihood (ML) criterion. Classification of strains into sublineages and informative genetic markers are shown. Support values correspond to bootstrap values

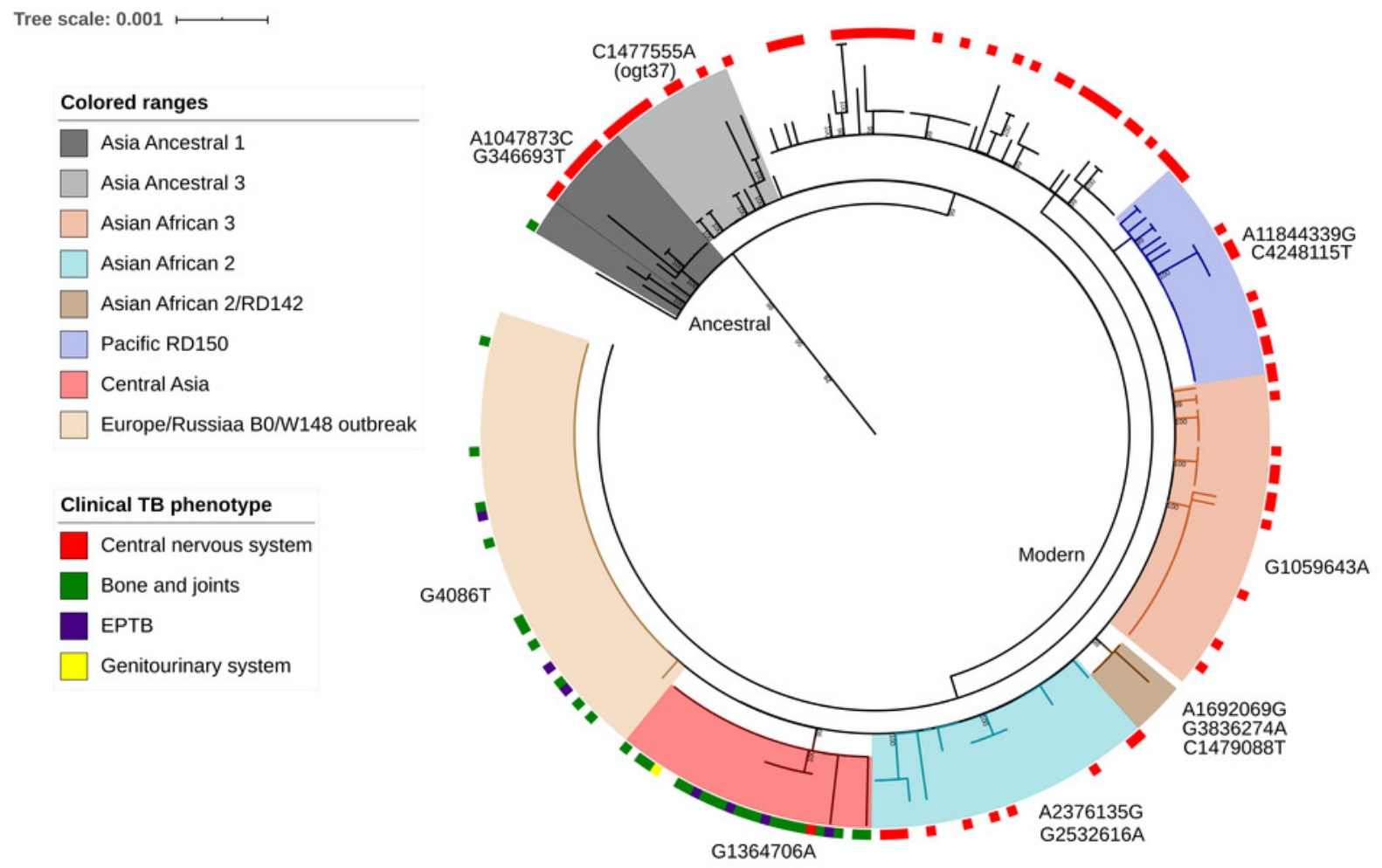

